\title{
How to reduce HIV risk among adolescent girls and young women in sub-Saharan Africa? Implementation science around the DREAMS Initiative
}

Population Council

Follow this and additional works at: https://knowledgecommons.popcouncil.org/departments_sbsr-hiv

Part of the Demography, Population, and Ecology Commons, Family, Life Course, and Society Commons, and the International Public Health Commons How does access to this work benefit you? Let us know!

\section{Recommended Citation}

Population Council. 2016. "How to reduce HIV risk among adolescent girls and young women in subSaharan Africa? Implementation science around the DREAMS Initiative," DREAMS Project Brief. Washington, DC: Population Council. 
Despite significant investment over the past decade to reduce HIV risk among adolescent girls and young women (AGYW), efforts to date have been relatively unsuccessful. Sex and age disparities in high-HIV burden sub-Saharan African countries remain almost unchanged and AIDS is now the leading cause of death among AGYW in the region. What is urgently needed is a shift in programming to better meet AGYW's needs, including addressing the structural issues predisposing AGYW to becoming infected with HIV.

The U.S. President's Emergency Plan for AIDS Relief (PEPFAR), the Bill \& Melinda Gates Foundation (BMGF), Girl Effect, Johnson \& Johnson, Gilead Sciences, and ViiV Healthcare are together supporting the Determined, Resilient, Empowered, AIDS-free, Mentored and Safe (DREAMS) Partnership in 10 sub-Saharan countries. DREAMS is delivering, through the public and private sector, a comprehensive package of evidence-based strategies that go beyond the health sector, addressing the structural drivers that fuel AGYW's HIV risk, including poverty, gender inequality, sexual violence, social isolation, and limited schooling.

The Population Council, with funding from BMGF and the U.S. Agency for International Development through Project SOAR, is leading the implementation science portfolio of this timely and innovative partnership, producing evidence to strengthen DREAMS activities and other community-based programing. The Council's research focuses on how best to reach the most vulnerable AGYW and their male partners and engage them in activities and services, and how to roll out preexposure prophylaxis (PrEP) for HIV prevention among AGYW.

By examining these critical HIV prevention issues, the Council's research will provide actionable evidence that implementers, policy makers, and others can use to improve how programs and services are delivered to vulnerable AGYW and their male partners.

\section{OUR RESEARCH}

The Population Council's implementation science portfolio examines different aspects of the DREAMS Partnership across diverse settings. The studies in our portfolio consist of formative research and program evaluation around the following themes:

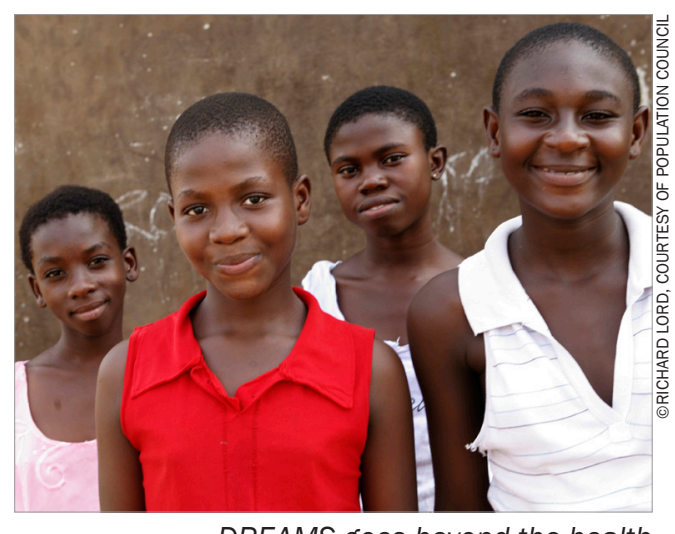

DREAMS goes beyond the health sector to reduce HIV risk.

The Population Council's 3-year implementation science work around the DREAMS Partnership began December 2015. The research is expected to begin yielding findings by the end of 2016. For more information, contact Sanyukta Mathur, Project Director, DREAMS Implementation Science Research, Population Council (smathur@popcouncil.org).

The Council is also building the capacity of DREAMS partners with support from the Girl Effect. For more information about these efforts, contact Miriam Temin, Project Director, Strengthening Capacity for DREAMS Girl Platforms (mtemin@popcouncil. org). 


\section{in} Identifying, linking, and retaining vulnerable AGYW in programs

DREAMS needs to know how best to find the most vulnerable AGYW, and link them to interventions that can reduce their risk of infection, such as HIV counseling and testing, conditional cash transfers for schooling, and social asset building. Our research in Kenya, Malawi, and Zambia will provide critical data for understanding:

1. How effective is community-based, girl-centered programming at:

- Reaching the most vulnerable AGYW?

- Connecting them to and sustaining their use of services?

- Reducing HIV-related risk factors?

2. What are the costs associated with comprehensive programming for HIV risk reduction?

\section{i}

\section{Reaching male partners of AGYW and} linking them to services

AGYW are vulnerable to HIV, in part due to the nature of their sexual partnerships and the characteristics of their male partners. Power differentials between AGYW and their partners may make it more difficult to negotiate condom use. Male partners often have more extensive sexual networks than AGYW. Comparatively, men have lower rates of using HIV services, including HIV testing and counseling and voluntary medical male circumcision, which may increase AGYW's HIV risk. Answering the following research questions in Malawi, South Africa, Swaziland, and Uganda will help DREAMS target AGYW's high-risk male partners and engage them in HIV services:

1. What are the profiles of male partners of AGYW in different "hot spots"?

2. How do male partners perceive the sexual dynamics between themselves and AGYW, and their HIV risk?

3. What programs successfully link male partners of AGYW with clinical HIV services? Are the "right" men being reached?

\section{WHAT IS IMPLEMENTATION SCIENCE IN THE CONTEXT OF DREAMS?}

Implementation science focuses on finding out what does and doesn't work, and using the results to strengthen programs and policies. We are using implementation science to:

- Diagnose service delivery barriers and facilitators.

- Assess the implementation process, outputs, and outcomes.

- Evaluate approaches for their effectiveness, cost, quality, and acceptability.

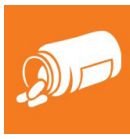

\section{Introducing PrEP among AGYW}

PrEP is a powerful HIV prevention tool that

has been shown to substantially reduce the risk of HIV infection for both men and women. Although information about the efficacy of PrEP is clearer for males than females, PrEP-in the context of a comprehensive HIV prevention program-may provide an important opportunity for AGYW to take control of their sexual health and reduce their HIV risk. Our studies in Tanzania and Uganda will contribute to a stronger evidence base by learning:

1. What are key considerations in introducing PrEP to AGYW (e.g., AGYW desires, health provider readiness, appropriateness for different subgroups of AGYW)?

2. What are the lessons from projects rolling out PrEP in "real world" settings?

3. What PrEP promotion strategies enhance adherence among AGYW?

\section{LOOKING AHEAD}

Our research findings will be used to guide the implementation of DREAMS, a bold partnership that has the potential to be a game changer for AGYW. The findings also have broader implications for global policy makers, program implementers, and donors by identifying strategies to better equip AGYW in preventing HIV infection.
POPULATION COUNCIL

Ideas. Evidence. Impact.
The Population Council confronts critical health and development issues-from stopping the spread of HIV to improving reproductive health and ensuring that young people lead full and productive lives. Through biomedical, social science and public health research in about 50 countries, the Council works with our partners to deliver solutions that lead to more effective policies, programs, and technologies to improve lives worldwide. Established in 1952 and headquartered in New York, the Council is a nongovernmental, nonprofit organization with an international board of trustees. popcouncil.org

Suggested citation: Population Council. 2016. "How to best reduce HIV risk among adolescent girls and young women in sub-Saharan Africa?: implementation science around the DREAMS Partnership,” DREAMS Project Brief. Washington, DC: Population Council. 